\title{
GORENSTEIN LOCAL HOMOMORPHISMS
}

\author{
LUCHEZAR L. AVRAMOV AND HANS-BJØRN FOXBY
}

\section{INTRODUCTION}

A Noetherian local ring is the algebraic version of a ring of germs of functions defined in neighborhoods of some point of an algebraic (or analytic) variety. Accordingly, local rings are naturally classified by the complexity of the singularity they describe, with the simplest class consisting of the regular rings, which correspond to nonsingular points. On the singular side a natural boundary is provided by the Cohen-Macaulay rings: beyond them pathological (that is, geometrically unpredictable) behavior becomes a common phenomenon.

During the last three decades much of the work in commutative algebra has concentrated on rings whose singularities interpolate between these two extremes. One of the most important developments early in that period was the discovery of the intermediate class of Gorenstein rings by Bass and Grothendieck. These authors demonstrated that Gorenstein rings provide a perfect framework for the investigation of duality phenomena, and this is the main reason behind their ubiquitous appearance in commutative algebra and algebraic geometry. They also noted that among the Gorenstein singularities one finds all local complete intersections, which describe points of transversal intersection of hypersurfaces.

The purpose of this note is to introduce some of the results of [2], where a relative theory of Gorenstein singularities is systematically developed. There are several aspects to our approach. First, it gives a unified treatment of hitherto unrelated relative Gorenstein notions, such as that of flat homomorphisms whose fibres are Gorenstein rings (Grothendieck), and surjective homomorphisms whose kernels are generated by regular sequences, or more generally, are Gorenstein ideals (Buchsbaum and Eisenbud). Next, it contains the theory of Gorenstein rings as the absolute case, that

Received by the editors May 18, 1989 and, in revised form, October 25, 1989.

1980 Mathematics Subject Classification (1985 Revision). Primary 13H10; Secondary 13D03, 13D25, 14M05. 
is, when one only considers homomorphisms from the ring of rational integers. Furthermore, it uncovers unsuspected properties of a large class of homomorphisms of Noetherian rings. Finally, it completes a step in a general program of extending the study of the different types of singularities referred to above from rings to their homomorphisms.

\section{BASS SERIES OF LOCAL DG RINGS}

The first step in our study of local Gorenstein homomorphisms consists in extending the definition of Bass numbers from modules over local rings to DG ( = differential graded) modules over local DG rings. We only consider DG rings which are (strictly skew) commutative and concentrated in nonnegative degrees, but make no a priori restrictions on the DG modules. A DG ring $R$ is called local, if $H_{0}(R)$ is a Noetherian local ring, and $H_{i}(R)$ is a finitely generated $H_{0}(R)$-module for $i \in \mathbf{Z}$. We set $\operatorname{fd} R=\sup \{i \in$ $\left.\mathbf{Z} \mid H_{i}(R) \neq 0\right\}$. There is a canonical augmentation $\varepsilon_{R}: R \rightarrow$ $H_{0}(R) \rightarrow \ell$ making the residue field $\ell$ of $H_{0}(R)$ a DG $R$ module. If $M$ is any DG $R$-module, we write $\mu_{R}^{i}(M)=$ $\operatorname{dim}_{\ell} \operatorname{Ext}_{R}^{i}(\not, M)$, as in the classical situation, but the Ext's are constructed-in the manner of Eilenberg and Moore-by use of appropriate resolutions in the DG category: for more details cf. [4]. We say that the Bass series $I_{R}^{M}(t)$ is defined, if $\mu_{R}^{i}(M)$ is finite for $i \in \mathbf{Z}$, and $\mu_{R}^{i}(M)=0$ for $i \ll 0$. When this holds then $I_{R}^{M}(t)=\sum_{i \in \mathrm{Z}} \mu_{R}^{i}(M) t^{i}$ is a formal Laurent series; we set $\mu_{R}^{i}=\mu_{R}^{i}(R)$ and $I_{R}(t)=I_{R}^{R}(t)$.

If $R$ is a local DG ring, $\widetilde{R}$ denotes the result of adjoining to $R$ exterior variables which kill a minimal set of generators of the maximal ideal of $H_{0}(R)$ (the cardinality of such a set is denoted DGedim $R$ ). If $R$ is a local ring, then $\widetilde{R}$ is a usual Koszul complex.

1.1. Proposition. Let $R$ be a local $D G$ ring, such that $\mathrm{fd} R<$ $\infty$. Then $\widetilde{R}$ is a local $D G$ ring, fd $\widetilde{R} \leq \mathrm{fd} R+\operatorname{DGedim} R, I_{R}(t)$ and $I_{\widetilde{R}}(t)$ both are defined, and are linked by the equality: $I_{R}(t)=$ $I_{\widetilde{R}}(t) t^{\text {DGedim } R}$.

By Bass [5], a local ring $R$ is Gorenstein if and only if $\mu_{R}^{i}=0$ for $i \neq \operatorname{depth} R$ and $\mu_{R}^{\text {depth } R}=1$. Now we extend the Gorenstein concept to the DG context: a local DG ring $R$ is called 
Gorenstein if $\mathrm{fd} R<\infty$ and $I_{R}(t)=t^{d}$ for some $d \in \mathbf{Z}$. As for rings, equivalent descriptions abound. Some of them are similar to characterizations of the "Gorenstein spaces" of [6].

1.2. Theorem. Let $R$ be a local $D G$ ring with $\mathrm{fd} R<\infty$. The following conditions are equivalent: (i) $R$ is Gorenstein; (ii) $I_{\widetilde{R}}(t)=$ $t^{-\mathrm{fd} \widetilde{R}}$; (iii) $I_{R}(t)$ is a Laurent polynomial; (iv) $H(\widetilde{R})$ is a Poincaré duality graded $/$-algebra.

It is well known that $I_{R}^{M}(t)$ is defined when $R$ is a local ring and $M$ is a complex of $R$-modules with $H(M)$ bounded above and $H_{i}(M)$ finitely generated over $R$ for $i \in \mathbf{Z}$, cf. [9, 7]. However, even if one is mainly interested in (complexes of) modules over local rings, local DG rings enter the picture as soon as one considers change-of-rings situations. For the rest of the note we consider a local homomorphism $\varphi:(R, \mathfrak{m}, \ell) \rightarrow(S, \mathfrak{n}, \ell)$. The (scheme-theoretic) fibre of $\varphi$ is then the local ring $S / \mathfrak{m} S$, but this yields no information of the homology of the $R$-module $S$. In order to take it into account, a fibre was constructed in [1] whichunless $\varphi$ is flat-is no more a local ring, but a local DG ring $F(\varphi) \rightarrow \ell$. It is defined uniquely up to homology isomorphism of augmented DG rings. By the general properties of the DG Ext functor, the vector spaces $\operatorname{Ext}_{F(\varphi)}^{i}(\ell, F(\varphi))$ are defined uniquely up to isomorphism. Furthermore, $H_{i}(F(\varphi)) \cong \operatorname{Tor}_{i}^{R}(k, S)$, hence $\mathrm{fd} F(\varphi)=\mathrm{fd}_{R} S$, the flat dimension of $S$ viewed as an $R$-module. Much of our work is based on the following result:

1.3. Theorem [4]. Let $\varphi$ be a local homomorphism such that $\mathrm{fd}_{R} S<\infty$, and let $M$ be a complex of $R$-modules with $H(M)$ bounded above. If $S \otimes_{=} M$ denotes the derived tensor product of complexes of $R$-modules, (cf. $[9,7])$ then there is an isomorphism of graded $\ell$-vector spaces:

$$
\operatorname{Ext}_{S}\left(\ell, S{\underset{=}{R}}_{R} M\right) \cong \operatorname{Ext}_{R}(\not, M) \otimes_{\ell} \operatorname{Ext}_{F(\varphi)}(\ell, F(\varphi)) .
$$

$S \otimes M$

In particular $I_{S}{ }^{=}(t)$ is defined precisely when $I_{R}^{M}(t)$ is, and then they are linked by the equality $I_{R}^{S \otimes} M(t)=I_{R}^{M}(t) I_{F(\varphi)}(t)$.

\section{LOCAL HOMOMORPHISMS}

A local homomorphism $\varphi$ is said to be Gorenstein if $\operatorname{fd}_{R} S$ is finite, and there is an integer $d$, such that $\mu_{R}^{i}=\mu_{S}^{i+d}$ for $i \in \mathbf{Z}$. 
(One sees that then necessarily $d=\operatorname{depth} S-\operatorname{depth} R$.) First we compare our notion of Gorenstein homomorphism with earlier ones, whenever these may be defined.

2.1. Proposition. (a) If $\varphi$ is flat, then $\varphi$ is Gorenstein if and only if $S / \mathrm{m} S$ is a Gorenstein ring. (b) If $\varphi$ is surjective and $\operatorname{pd}_{R} S<$ $\infty$, then $\varphi$ is Gorenstein if and only if $\operatorname{Ker} \varphi$ is a Gorenstein ideal. (c) If $\varphi$ is the structure homomorphism $\mathbf{Z}_{(p)} \rightarrow S, p=\operatorname{char} \ell$, then $\varphi$ is Gorenstein if and only if $S$ is a Gorenstein ring.

Sketch of proof. (a) When $\varphi$ is flat, the claim follows from the equality of formal Laurent series $I_{S}(t)=I_{R}(t) I_{S / \mathrm{m} S}(t)$, which is proved in [8] (this is a particular case of (1.3): set $M=R$ and note that if $\varphi$ is flat, there is a homology isomorphism $F(\varphi) \rightarrow$ $S / \mathfrak{m} S$ ). (b) If $\operatorname{Ker} \varphi$ is a Gorenstein ideal, then the standard change of rings spectral sequence ${ }_{2} E^{p q}=\operatorname{Ext}_{S}^{p}\left(\not R, \operatorname{Ext}_{R}^{q}(S, R)\right) \Rightarrow$ $\operatorname{Ext}_{R}^{p+q}(\not{k}, R)$ degenerates into isomorphisms $\operatorname{Ext}_{S}^{p}(\not{k}, S) \cong$ $\operatorname{Ext}_{R}^{p+g}(\not R, R), g=\operatorname{grade}_{R} S$. For the converse one uses the fact that $H(F(\varphi))=\operatorname{Tor}^{R}(\mathcal{E}, S)$ has Poincaré duality, cf. 1.2 , in order to show that the minimal free resolution of the $R$-module $S$ is self-dual. (c) is clear, since $\mathbf{Z}_{(p)}$ has finite global dimension.

Next we comment on the ubiquity of Gorenstein homomorphisms.

2.2. Theorem. Let $\varphi: R \rightarrow S$ be a local homomorphism, such that $\operatorname{fd}_{R} S<\infty$. The following conditions are then equivalent: (i) $\varphi$ is Gorenstein; (ii) the local DG ring $F(\varphi)$ is Gorenstein; (iii) $\mu_{R}^{i}(M)=\mu_{S}^{i+d}\left(S \otimes_{R} M\right)$ for all $i$, some $d$ and some flat $R$-module with $\operatorname{Ext}_{R}(\mathcal{K}, M) \neq 0$; (iv) $\mu_{R}^{i+\operatorname{depth} R}(M)=\mu_{S}^{i+\operatorname{depth} S}\left(S \otimes_{R} M\right)$ for all $i$ and each complex of $R$-modules $M$, such that $H(M)$ is bounded above; (v) $\operatorname{id}_{S}(S \underset{=}{\otimes} M)<\infty$ for each homologically bounded complex of $R$-modules $M$, with $\operatorname{id}_{R} M<\infty$ and $H_{i}(M)$ finitely generated for all $i$; (vi) $\operatorname{id}_{S}\left(S \otimes_{=} M\right)<\infty$ for some complex $M$ of $R$-modules, such that $\operatorname{Ext}_{R}(\mathscr{R}, M) \neq 0$.

Gorenstein local homomorphisms form a remarkably rigid class:

2.3. Proposition. (a) $\varphi$ is Gorenstein if and only if $\hat{\varphi}: \widehat{R} \rightarrow \widehat{S}$ is Gorenstein. (b) If $\psi: Q \rightarrow R$ and $\varphi: R \rightarrow S$ are local homomorphisms with $\mathrm{fd}_{Q} R<\infty$ and $\mathrm{fd}_{R} S<\infty$, then $\varphi \psi$ is Gorenstein if and only if $\varphi$ and $\psi$ are. 
Proof. (a) follows from the standard: $\mathrm{fd}_{R} S=\mathrm{fd}_{\widehat{R}} \widehat{S}, I_{R}(t)=$ $I_{\widehat{R}}(t)$, and $I_{S}(t)=I_{\widehat{S}}(t)$. (b) is implied by the double inequalities $\mu_{Q}^{i+\operatorname{depth} Q} \leq \mu_{R}^{i+\operatorname{depth} R} \leq \mu_{S}^{i+\operatorname{depth} S}, i \in \mathbf{Z}$, which are immediate from (1.3).

Gorenstein homomorphisms appear in a natural way when one looks for conditions under which a map of rings transfers the Gorenstein property between them. Thus we can complete some results from [3].

2.4. Theorem. For a local homomorphism $\varphi: R \rightarrow S$ the following conditions are equivalent: (i) $R$ is a Gorenstein and $\varphi$ is Gorenstein. (ii) $S$ Gorenstein and $\mathrm{fd}_{R} S<\infty$; (iii) $S$ is Gorenstein and $\mathrm{id}_{R} S<\infty$.

Sketch of proof. That (ii) $\Rightarrow$ (i) follows readily from (1.3). For (i) $\Rightarrow$ (iii) one uses (1.3) to see that $S$ is a Gorenstein ring, and concludes that $\operatorname{id}_{R} S<\infty$ by the equivalence of the finiteness of the flat or injective dimension over the Gorenstein ring $R$. For (iii) $\Rightarrow$ (ii) consider $M=\operatorname{Hom}_{R}(S, E)$ where $E$ is the injective envelope of the $R$-module $\mathscr{k}$. One proves that $\operatorname{Tor}^{R}(\mathcal{K}, M) \neq 0$, $\mathrm{fd}_{R} M<\infty$, and $\operatorname{id}_{R} M<\infty$ : It follows from [7] that $R$ is Gorenstein, hence $\operatorname{fd}_{R} S<\infty$ as above.

\section{DuAlizing COMPleXes}

For our purpose, a (normalized) dualizing complex is a homologically bounded complex of $R$-modules $D$, with finitely generated homology, and such that $I_{R}^{D}(t)=t^{n}$ for some $n \in \mathbf{Z}$ (for $n=\operatorname{dim} R)$. The behavior of dualizing complexes under extension of scalars is an important and delicate question, which we now address. Part of our results needs mild technical restrictions. We say $\varphi$ satisfies a standard condition if one of the following holds: (a) $\varphi$ is flat; (b) $\varphi$ is essentially of finite type; (c) $\ell \rightarrow \ell$ is a separable field extension; (d) $R$ is equicharacteristic.

3.1. Theorem. Let $D$ be a complex of $R$-modules such that $H(D)$ is bounded above and $H_{i}(D)$ is finitely generated for $i \in \mathbf{Z}$. The following are then equivalent (if $\varphi$ satisfies a standard condition): (i) $D$ is a (normalized) dualizing complex of $R$ and $\varphi$ is Gorenstein; (ii) $S \otimes_{R} D$ is a (normalized) dualizing complex of $S$ and $\mathrm{fd}_{R} S<\infty$.

Proof. The equivalence of the unbracketed statements is derived directly from (1.3) and from the corresponding definitions. From 
(1.3) one also gets the equality $\mathrm{DG} \operatorname{depth}_{S} S \underset{=}{\otimes} D-\operatorname{depth} S=$ DG depth ${ }_{R} D$-depth $R$, where DG depth ${ }_{R} M$ denotes the order of $I_{R}^{M}(t)$. Noting that $D$ is normalized if and only if $D G \operatorname{depth}_{R} D-$ $\operatorname{dim} R=0$, apply the next result.

3.2. Theorem. (a) If $\varphi$ is Gorenstein, then $\operatorname{dim} R-\operatorname{depth} R \geq$ $\operatorname{dim} S-\operatorname{depth} S$. (b) If $\operatorname{fd}_{R} S<\infty$ and $\varphi$ satisfies a standard condition, then $\operatorname{dim} R-\operatorname{depth} R \leq \operatorname{dim} S-\operatorname{depth} S$.

The last theorem implies, in particular, that if $\varphi$ is Gorenstein, then $S$ is Cohen-Macaulay if and (under a special condition) only if $R$ is: Compare (2.4) for the Gorenstein property. Finally, we note for Gorenstein homomorphisms the following localization property, whose proof heavily depends on dualizing complexes, and on (3.1) and (2.3) above.

3.3. Theorem. Assume that the formal fibres of $R$ are Gorenstein. If $\varphi$ is a Gorenstein homomorphism, then for each prime ideal $\mathfrak{q}$ of $S$ the induced homomorphism $R_{\mathrm{q} \cap R} \rightarrow S_{\mathrm{q}}$ is Gorenstein, and the formal fibres of $S$ are Gorenstein.

\section{REFERENCES}

1. L. L. Avramov, Local algebra and rational homotopy, Astérisque 113-114 (1984), 15-43.

2. L. L. Avramov and H.-B. Foxby, Locally Gorenstein homomorphisms, Københavns Univ. Mat. Inst. Preprint Series no. 16, 1988.

3. L. L. Avramov, H.-B. Foxby and S. Halperin, Descent and ascent of local properties along homomorphisms of finite flat dimension, J. Pure Appl. Algebra 38 (1985), 167-185.

4. L. L. Avramov, H.-B. Foxby and J. Lescot, Bass series of local ring homomorphisms of finite flat dimension, Københavns Univ. Mat. Inst. Preprint Series no. 15, 1988.

5. H. Bass, On the ubiquity of Gorenstein rings, Math. Z. 82 (1963), 8-28.

6. Y. Félix, S. Halperin and J.-C. Thomas, Gorenstein spaces, Adv. Math. 71 (1988), 92-112.

7. H.-B. Foxby, $A$ homological theory of complexes of modules, Københavns Univ. Mat. Inst. Preprint Series no. 19, 1981.

8. H.-B. Foxby and A. Thorup, Minimal injective resolutions under flat base change, Proc. Amer. Math. Soc. 67 (1977), 27-31.

9. R. Hartshorne, Residues and duality, Lecture Notes in Math., 20, SpringerVerlag, Berlin, 1966.

Institute of Mathematics, Ul. "Akad. G. Bončev' No. 8, 1113 Sofia, BULGARIA

Matematisk Institut, Universitetsparken 5, 2100 Kiobenhavn $\varnothing$, DeNMARK 